\section{Karen Midthun}

Karen Midthun, the Director of the FDA's Center for Biologics Evaluation and Research (CBER), is retiring in January. An infectious disease doctor by training, she has spent the past 22 years at the agency regulating vaccines, cellular and gene therapies, blood products and more. She spoke with Asher Mullard about her achievements in her long career at the agency and the regulatory challenges her successor will face.

\section{Q What are your proudest achievements at the FDA?}

The Center has been able to do so much during my tenure here. But I'd point to some of our important approvals, our public health mission and then our work on policy development.

We have approved many influenza vaccines over the past decades, including novel technologies that have really improved our manufacturing base for influenza vaccines. We've also approved the first adjuvant influenza vaccine, which was actually an $\mathrm{H} 5 \mathrm{~N} 1$ vaccine for potential pandemic response. And we've approved vaccines that have had a huge public health impact, including vaccines for pneumococcal conjugate disease, meningococcal diseases and human papilloma virus.

We also regulate products for rare diseases, and have approved biologics including recombinant proteins technology to treat haemophilia A and B and some very rare clotting disorders. We've approved six cord blood products that are used to treat disorders of the haematopoietic system. And, we have approved some innovative therapies, including most recently an oncolytic virus for melanoma. This is really just a snapshot of our product approvals.

Clearly these vaccine approvals have been a huge part of our public health mission. Another success was our work in 2009 on the pandemic response. We put a huge amount of work into trying to develop vaccine reagents, and worked with our advisory committee to come up with a rapid pathway to be able to license these monovalent $\mathrm{H} 1 \mathrm{~N} 1$ vaccines.

A third area of success for us has been our engagement in developing important policies. First and foremost, we set up the framework for the regulation of human cell and tissue products. We've also worked very closely with the Center for Drug Evaluation and Research (CDER) to develop policy for biosimilars.
Q In terms of vaccine approvals, what regulatory challenges will your successor face?

In the vaccine area, some of the low-hanging fruit has been picked. In many cases drug developers have already developed the vaccines that prevent the infectious diseases that are more common. So now people are looking to develop vaccines against very rare infections. And this presents different challenges than when you are developing a drug for a rare disease. With rare infections, you have no idea who is going to get sick. To give you a flavour of this, for a pneumococcal conjugate vaccine that we approved in the early 2000s, the drug developers had to vaccinate around 40,000 infants to get 17 cases of infection. Fortunately the case split was 17 to 0 when they unblinded the data, so the effectiveness was compelling. But, compared with other infections that was a common disease. That gives you the sense that you just can't do the kind of effectiveness studies that we have been using to demonstrate efficacy. We need to figure out if there is something

Vaccinating pregnant mothers to provide protection to infants is another great area of opportunity. We had a vaccines advisory committee in November to discuss this area of science. And whereas for a long time there just didn't seem to be interest from vaccine developers in this space, there is now a lot of interest in it. We are very excited about this, but obviously there are a lot of things that still need to be worked out.

\section{Q Gene therapies have made a resurgence} during your tenure. What are the remaining challenges here?

We have huge activity in this area, so I'm really hoping that with the body of science that has been developed we will start seeing more of these products. I think that a lot of understanding has been gained since the else that we can use instead.

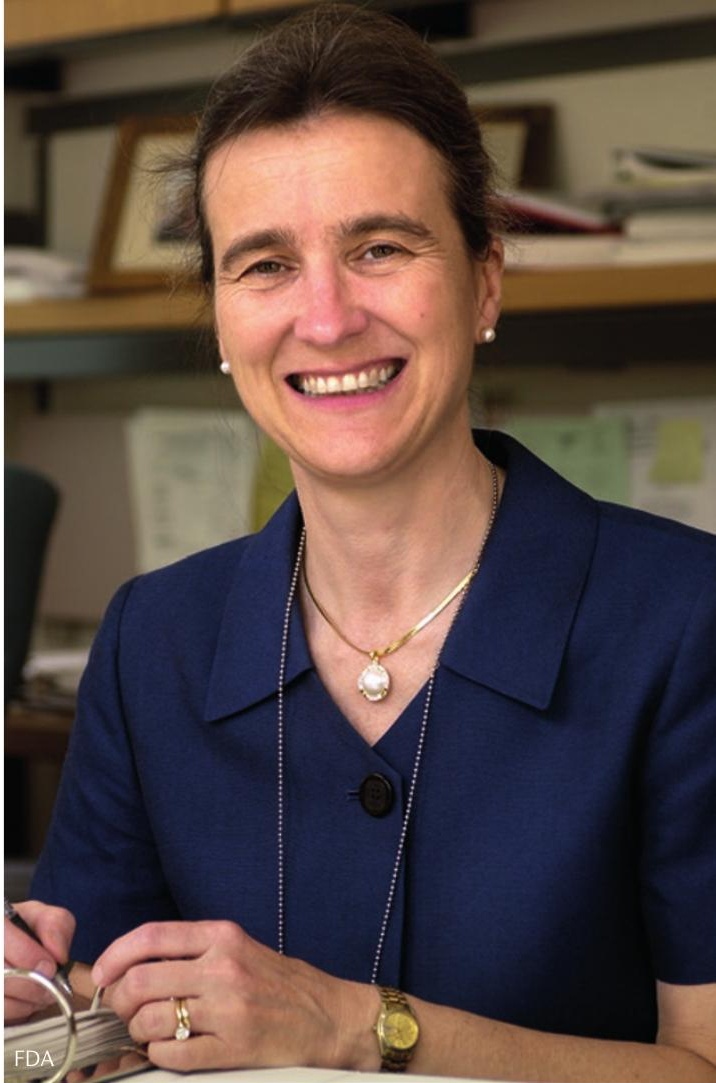

Jesse Gelsinger incident, not only in terms of how to run clinical trials but also in terms of product characterization and what kind of vectors we can use. I'd really like to think that we are now with gene therapies where we were 25 years ago with some of the monoclonal antibodies.

Q In terms of your work on the biosimilar policy, a lot of interest in this pathway is with regard to the approval of biosimilar antibody drugs, which are regulated by CDER. What's your interest in this space? The biosimilar policy applies to all biologic products. I think we recognize that it can be daunting for drug companies to show that their products are highly similar to a reference product, and it is much easier to do for well-characterized somewhat simpler proteins. As you can imagine, many of the products CBER regulates are incredibly complex. But, let's put it this way: 10-15 years ago, people probably could not have imagined having the analytical technology to show biosimilarity to a reference therapeutic antibody. We need to expect that as the science advances, we may find ourselves in the situation where some of the products CBER regulates may lend themselves to this pathway as well.

\section{Q What's next for you?}

I don't know. I do look forward to having more personal time. But at heart I'm a vaccinologist and I hope to still be able to contribute to the community. 